\title{
1 Four layer multi-omics reveals molecular 2 responses to aneuploidy in Leishmania
}

3 Cuypers B. ${ }^{1,2}$, Meysman P. ${ }^{2}$, Erb I. ${ }^{3}$, Bittremieux W. ${ }^{4}$, Valkenborg D. ${ }^{5,6}$, Baggerman G. ${ }^{6,7}$, Mertens I. ${ }^{6,7}$,

4 Shyam Sundar ${ }^{8}$, Basudha Khanal ${ }^{9}$, Notredame C. ${ }^{3}$, Dujardin, J-C. ${ }^{1,10 \#}$, Domagalska M.A. ${ }^{{ }^{*} \#}$, Laukens K. ${ }^{2^{*} \#}$

5 1. Molecular Parasitology Unit, Department of Biomedical Sciences, Institute of Tropical Medicine,

6 Antwerp, Belgium.

3. Centre for Genomic Regulation (CRG), The Barcelona Centre of Science and Technology, Barcelona, Spain.

4. Skaggs School of Pharmacy and Pharmaceutical Sciences, University of California San Diego, La Jolla, CA 92093, USA.

5. Center for Statistics - University of Hasselt, Hasselt, Belgium.

6. Center for Proteomics, University of Antwerp, Antwerp, Belgium.

7. VITO, Mol, Belgium.

8. Institute of Medical Sciences, Banaras Hindu University, Varanasi, India

9. BP Koirala Institute of Health Sciences, Dharan, Nepal

* These authors share senior authorship

\# Corresponding authors 


\section{Abstract}

23

Aneuploidy causes system-wide disruptions in the stochiometric balances of transcripts, proteins, and metabolites, often resulting in detrimental effects for the organism. The protozoan parasite Leishmania has an unusually high tolerance for aneuploidy, but its molecular consequences remain poorly understood. Here, we present the first integrated analysis of the genome, transcriptome, proteome, and metabolome of highly aneuploid Leishmania donovani strains. Our analysis unambiguously establishes that, despite this tolerance, aneuploidy in Leishmania globally and proportionally impacts the transcriptome and proteome of affected chromosomes, ultimately explaining the degree of metabolic differences between strains. This impact was present in both proliferative and infectious life stages. Protein complex subunits, secreted proteins, and noncytoplasmic proteins, responded less or even not at all to aneuploidy-induced dosage changes. In contrast to other Eukaryotes, widespread transcript regulation modulating aneuploidy-related stress was absent. Further, the majority of regulated proteins was encoded by non-aneuploid chromosomes and had no underlying transcript change, suggesting that aneuploidy induces extensive posttranscriptional protein-level modulation. This makes Leishmania a unique Eukaryotic model for elucidating post-transcriptional protein-abundance modulation in the context of aneuploidy. 


\section{Introduction}

Aneuploidy is the presence of an abnormal number of chromosomes in a cell. All genes encoded by aneuploid chromosomes undergo a shift in their copy number or "gene dosage". This large number of unbalanced dosage changes typically leads to broad-scale disruptions in the stoichiometric balances of transcripts, proteins, and metabolites, making aneuploidy most widely known for its detrimental effects. Indeed, aneuploidy can cause oxidative stress, energetic stress, misfolded protein stress (proteotoxicity), and metabolic stress at the cellular level ${ }^{1,2}$. In multicellular organisms, where cells are closely fine-tuned to work together, this often leads to growth delays or lethality ${ }^{1}$.

Contrastingly, aneuploidy can also result in fitness gains. In fungi, specific karyotypes confer resistance against drugs, toxicants, or increase survival under nutrient limiting conditions ${ }^{3-6}$. Likewise, in cancer cells, aneuploidy can be tumor growth-promoting ${ }^{7}$. By altering the copy number of many genes at once, aneuploidy enables cells to explore a wide fitness landscape ${ }^{8}$. Particularly when facing very novel or competitive conditions, aneuploidy might allow the rapid evaluation of many divergent phenotypes ${ }^{9}$. Fully understanding this complex trade-off between aneuploidy fitness gains and losses requires a systems biological comprehension of its molecular impact.

In this context, an organism with a remarkably high tolerance for aneuploidy is the protozoan parasite Leishmania (Euglenozoa, Kinetoplastida). Transmitted by its sand-fly vector, Leishmania causes the disease leishmaniasis in humans and various vertebrate animals. The parasite displays exceptional genome plasticity in experimental conditions. A genomic analysis of 204 in vitro cultured Leishmania donovani strains uncovered up to 22 aneuploid chromosomes (out of 36) within a single strain ${ }^{10}$. Moreover, all 36 chromosomes have the capacity to become aneuploid and lead to viable parasites ${ }^{11}$. Strikingly, parasite populations do not have uniform karyotypes but display a mosaic of them. Singlecell sequencing of 1560 L. donovani parasites from a clonal population identified 128 different karyotypes, of which four were dominant ${ }^{12}$. This genomic instability and mosaicism could explain the parasite's ability to rapidly select more advantageous karyotypes upon encountering novel in vivo or in vitro environments ${ }^{13}$.

Leishmania also features an unusual gene expression system that is markedly different from most other Eukaryotes. Transcription of protein-coding genes cannot be controlled individually as these lack separate promoters. Instead, they are transcribed constitutively in long polycistronic units that span 10 's to 100 's of functionally unrelated genes ${ }^{14}$. The abundance of individual mRNAs and proteins is 
still regulated, but entirely post-transcriptionally ${ }^{13,15}$. Thus, the most straightforward evolutionary path towards altered gene expression might be altered gene dosage, which could explain the high incidence of aneuploidy ${ }^{13}$. While aneuploidy in Leishmania is known to directly affect the transcription of encoded transcripts, its molecular impact on the proteome and metabolome remains poorly understood $^{13,16}$.

Altogether, Leishmania provides a unique perspective into the molecular impact of aneuploidy as (1) a system where transcriptional control of individual protein-coding genes is absent, and as (2) an organism with a remarkably high aneuploidy tolerance. Here we present the first study that generated and integrated genome, transcriptome, proteome, and metabolome data of highly aneuploid $L$. donovani strains. This multi-omics analysis unambiguously demonstrates that aneuploidy in Leishmania globally and drastically impacts the transcriptome and proteome of affected chromosomes, ultimately explaining the degree of metabolic differences between strains. This impact appears to be present throughout the parasite's life cycle as we observed it in both the parasite's proliferative and infectious life stages. Interestingly, not all proteins responded equally to dosage changes; through dosage compensation, particular proteins such as protein complex subunits, secreted proteins, and non-cytoplasmic proteins responded less or even not at all to aneuploidy. Interestingly, we did not observe the widespread regulation at the transcript level that typically modulates the effects of aneuploidy in other Eukaryotes. Instead, Leishmania features a surprisingly high number of regulated proteins from non-aneuploid chromosomes, which are likely posttranscriptionally modulated. 
89

90

91

92

93

94

95

96

97

98

99

100

101

102

103

104

105

106

107

108

109

110

111

112

113

114

115

116

117

118

\section{Methods}

\section{Parasites}

The experiments in this study were performed on six cloned strains of Leishmania donovani: BPK275/0 cl18 (BPK275), BPK173/0 cl3 (BPK173), BPK282 cl4 (BPK282), BPK288/0 cl7 (BPK288), BPK178/0 cl3 (BPK178), BHU575/0 cl2 (BHU575) (Supplementary Table S1.A). A strain is a parasite population derived by serial passage in vitro from a primary isolate (from patient) with some degree of characterization, in our case bulk genome sequencing (definition modified from ${ }^{17}$ ). These strains were cloned to ensure genomic homogeneity. Parasites were cultured in HOMEM (Gibco) supplemented with $20 \%$ heat-inactivated fetal bovine serum (Biochrom), three days for replicative forms (LOG growth phase, 4-6 replicates) and six days for non-replicative forms (STAT, 4 replicates). Parasites from all replicates were washed twice by centrifugation at $18,000 \times \mathrm{g}$ at $0^{\circ} \mathrm{C}$, resuspension in ice-cold PBS buffer (PBS; pH 7.4 - Invitrogen), and pelleted at $18,000 \times \mathrm{g}$ at $0{ }^{\circ} \mathrm{C}$. DNA, RNA, protein, and metabolite extractions were carried out in parallel (see further).

\section{DNA/RNA extraction, library preparation, and sequencing}

DNA and RNA were extracted from the parasite pellets with the AllPrep DNA/RNA Mini Kit (Qiagen). The RNA integrity was then verified with the RNA 6000 Nano Kit using a Bioanalyzer 2100 (Agilent). DNA was quantified with the Qubit dsDNA BR assay (Thermo Fisher Scientific) and RNA with the Qubit RNA HS Assay.

Library preparation and sequencing of the DNA samples were performed at the Beijing Genomics Institute (BGI). Libraries were prepared with the TruSeq Nano DNA HT sample prep kit (Illumina) and 2 x 151 bp sequenced on the Illumina HiSeq 4000 platform. RNA sequencing libraries were prepared using the Spliced leader sequencing (SL-Seq) method as described in Cuypers et al. (2017) ${ }^{15}$. This protocol makes use of the presence of the affixed 39 nucleotide sequence spliced-leader (SL) that is present at the 5' end of all functional Leishmania mRNAs. RNAs containing a SL are selectively amplified with the protocol, and adapters required for Illumina sequencing are ligated. The SL-Seq libraries were $1 \times 50$ bp sequenced with the HiSeq 1500 platform of the Center of Medical Genetics Antwerp (Belgium).

\section{Genome and Transcriptome data analysis}

The quality of the raw sequencing data was verified with FASTQC v0.11.4 ${ }^{18}$. Reads were quality trimmed using Trimmomatic v035 19 with the settings 'LEADING:20 TRAILING:20 
ILLUMINACLIP:adapter.fa:2:40:15 SLIDINGWINDOW:4:20 MINLEN:36'. Reads were subsequently aligned to the L. donovani LdBPKv2 ${ }^{20}$ reference genome using BWA Mem version 0.7.15 ${ }^{21}$ with default parameters. The resulting bam files were sorted and indexed with samtools ${ }^{22}$. Mapping reports were generated using samtools flagstat.

Somy estimations and gene copy numbers were calculated as described in Downing (2011) ${ }^{23}$. Briefly, the somy value of a chromosome (S) was calculated by dividing its median sequencing depth (i.e. the number of times a nucleotide is read during sequencing, $d_{c h}$ ) by the median $d_{c h}$ of all 36 chromosomes $\left(d_{m c h}\right)$ and multiplying this value by two (for a predominantly diploid organism). Gene copy number per haploid genome (d) was defined as a raw depth for that gene $\left(d_{r}\right)$, divided by the median depth of its chromosome $\left(d_{c h}\right)$ : so that $d=d_{r} / d_{c h}$. Gene copy number estimations per cell (full depth, gene dosage) were then calculated by multiplying $d$ with S. SNPs were previously characterized in ${ }^{10}$. RNA-Seq gene read count tables were generated with the HTSeq tool version $0.6 .1^{24}$. Count data were normalized for library size by dividing each transcript count by the geometric mean of all counts for that library ${ }^{25}$. Differential gene expression between LOG and STAT life stages was calculated with DESeq2 v1.30.126.

\section{Proteomics}

\section{Sample preparation}

Cell pellets were efficiently lysed using $200 \mu$ I RIPA buffer and 1x HALT protease inhibitor (Thermo Scientific), combined with a $30 \mathrm{sec}$ sonication (Branson Sonifier SLPe ultrasonic homogenizer, Labequip, Ontario, Canada) with an amplitude of $50 \%$ on ice. After centrifugation of the samples for $15 \mathrm{~min}$ at $10,000 \mathrm{~g}$ at $4{ }^{\circ} \mathrm{C}$, the cell pellet was discarded. Next, the protein concentration was determined using the Pierce BCA protein Assay kit in combination with a NanoDrop 2000 photospectrometer (Thermo Scientific).

For each sample, $25 \mu \mathrm{g}$ of proteins were reduced using $2 \mu \mathrm{l}$ of $200 \mathrm{mM}$ tris(2-carboxyethyl) phosphine, in a volume of $20 \mu \mathrm{l} 200 \mathrm{mM}$ triethylammonium bicarbonate (TEAB), and incubated for $1 \mathrm{~h}$ at $55^{\circ} \mathrm{C}$. After alkylation of the proteins with $2 \mu \mathrm{L}$ of $375 \mathrm{mM}$ iodoacetamide for $30 \mathrm{~min}$ protected from light, 6 volumes of ice-cold acetone were added, and the samples were incubated overnight at $-20^{\circ} \mathrm{C}$. The next day, samples were centrifuged for $10 \mathrm{~min}$ at $10.000 \mathrm{~g}$ at $4^{\circ} \mathrm{C}$, the acetone was removed, and pellets were resolved in $20 \mu \mathrm{l}$ of $200 \mathrm{mM}$ TEAB. Proteins were then digested with trypsin (Promega) overnight at $37^{\circ} \mathrm{C}$ with an enzyme trypsin ratio of $1: 50$. Before LC-MS/MS analysis, the samples were desalted with Pierce C18 spin columns according to the manufacturer's instructions (Thermo Scientific). 
Each of the digested samples was separated by nano reverse phase C18 (RP-C18) chromatography on an Easy-nLC 1000 system (Thermo Scientific, San Jose, CA) using an Acclaim C18 PepMap ${ }^{\circledR} 100$ column (75 $\mu \mathrm{m} \times 2 \mathrm{~cm}, 3 \mu \mathrm{m}$ particle size) connected to an Acclaim PepMap ${ }^{\text {TM }}$ RSLC C18 analytical column (50 $\mu \mathrm{m} \times 15 \mathrm{~cm}, 2 \mu \mathrm{m}$ particle size) (Thermo Scientific, San Jose, CA). Of each sample, a total of $1 \mu \mathrm{g}$ of peptides were loaded on the column. Before loading, digests were dissolved in mobile phase $A$, containing $2 \%$ acetonitrile and $0.1 \%$ formic acid, at a concentration of $1 \mu \mathrm{g} / 10 \mu \mathrm{L}$ and spiked with 20 fmol Glu-1-fibrinopeptide B (Glu-fib, Protea biosciences, Morgantown, WV). A linear gradient of mobile phase B (0.1\% formic acid in $100 \%$ acetonitrile) from 0 to $45 \%$ in $90 \mathrm{~min}$, followed by a steep increase to $100 \%$ mobile phase $B$ in $10 \mathrm{~min}$, was used at a flow rate of $300 \mathrm{~nL} / \mathrm{min}$. Liquid Chromatography was followed by MS (LC-MS/MS) and was performed on a Q-Exactive Plus mass spectrometer equipped with a nanospray ion source (Thermo Fisher, Waltham, MA, USA). The high-resolution mass spectrometer was set up in an MS/MS mode where a full scan spectrum $(350-1850 \mathrm{~m} / \mathrm{z}$, resolution 70,000 ) was followed by a high energy collision activated dissociation (HCD) tandem mass spectra (100 $-2000 \mathrm{~m} / \mathrm{z}$, resolution 17,500). Peptide ions were selected for further interrogation by tandem MS as the twenty most intense peaks of a full scan mass spectrum. The normalized collision energy used was set at $28 \%$. A dynamic exclusion list of $20 \mathrm{sec}$ for the data-dependent acquisition was applied.

\section{Proteomic Data Analysis}

167 Thermo raw files were converted to mzML files using MSConvert v3.0. Label-free protein quantification 168 (LFQ) was carried out with MaxQuant version 1.6.0.16 ${ }^{27}$ using the following settings (other settings 169 were kept default): Oxidation (M) and acetyl (Protein N-term) were indicated as variable modifications, 170 carbamidomethyl (C) was indicated as a fixed modification, digestion with trypsin with maximum two missed cleavages, match between runs $=$ yes, dependent peptides $=$ yes. The search database was the LdBPKV2 proteome previously published by our group ${ }^{13}$, and reversed proteins were used as decoys.

\section{Metabolomics}

\section{Sample preparation}

175 Sample pellets were extracted using a 1:3:1 (v:v:v) chloroform/methanol/water extraction, subjected 176 to liquid chromatography using a $2.1 \mathrm{~mm}$ ZIC-HILIC column (Sequant), and analyzed using the Orbitrap 177 Exactive (Thermo Fisher Scientific) platform at the Glasgow Polyomics Center (University of Glasgow, 178 Glasgow, Scotland) exactly as described elsewhere ${ }^{28,29}$. Both negative and positive ion modes were 
run in parallel with rapid polarity switching. Samples were analyzed in randomized order and the same analytical batch. Additionally, to aid accurate metabolite identification, verify LC-MS stability, and identify contaminants, the following control samples were included as well: (1) solvents blanks, (2) serial dilutions of a pooled sample (Undiluted, 1/2, 1/4, 1/8, and 1/16), (3) Authentic standard mixes containing in total 217 metabolites $(50-400 \mathrm{Da})$ and representing a wide array of metabolic classes and pathways, (4) An amino acid standard mix (Sigma Product No. A9906).

\section{Metabolomic data analysis}

Data preprocessing was carried out with the XCMS 1.42.0 ${ }^{30}$ and mzMatch 1.0.1 ${ }^{31}$ packages in $\mathrm{R}$, exactly as described elsewhere ${ }^{32}$. Briefly, raw spectra (mzXML format) were first subjected to retention time alignment with ObiWarp ${ }^{33}$, after which peak detection was carried out with the centWave algorithm ${ }^{34}$. Peaks were then filtered: 1) Corresponding peaks from the four biological replicates were allowed a maximum reproducibility standard deviation (RSD) of $0.5,2$ ) Peak shape (CoDA-DW $>0.8), 3$ ) Detection in at least 3 out of 4 replicates of a single strain and 4) Minimal peak intensity was set to 3000 . The peaks were putatively identified using sequentially the LeishCyc database ${ }^{35}$, LIPID MAPS ${ }^{36}$, KEGG ${ }^{37}$, the peptide database included in mzMatch, and the Human Metabolome Database ${ }^{38}$. Finally, the filtered peaks were normalized for total ion count and subjected to manual quality screening.

\section{Integrated multi -omic analysis}

For each comparison between two strains, we calculated the $\log _{2}$ fold change ( $\log _{2} \mathrm{FC}$ ) for genes (gene dosage), transcripts, proteins and metabolites. These $\log _{2} \mathrm{FC}$ values were obtained by taking the $\log _{2}$ ratio of the average abundance between two strains. Associated $p$-values were obtained by performing a Student $t$-test for each comparison and subsequently correcting it with the Benjamini-Hochberg algorithm to limit the False Discovery Rate (FDR) to 5\%. A Log2FC cutoff of 1 and adjusted $p$-value cutoff of 0.05 was used for all 'omic layers, but for gene dosage, an additional criterium was applied; only copy number differences of at least 0.5 /haploid genome were considered as biologically significant ${ }^{39}$. All plots were generated using the ggplot v 2_2.1.0 package ${ }^{40}$, except the Circos plots which were created with Circos v0.69 ${ }^{41}$. Gene ontology enrichments were calculated with the Fisher Exact test using Python 3.8.5 and the matplotlib v3.3.4 ${ }^{42}$ and SciPy v1.6.1 ${ }^{43}$ libraries. GO annotations were obtained from the gene ontology consortium (http://www.geneontology.org/). 


\section{Dosage compensation analysis}

209 The following protein features were checked for their relation with protein dosage compensation:

210 degree (i.e., number of protein interactions as transferred from STRING), interacting (yes/no), stress

211 centrality (number of shortest paths between all node pairs in the protein-protein interaction network,

212 that pass through the specific protein), length (as a proxy for transcript and protein molecular size),

213 GRAVY (grand average of hydropathy), solubility (soluble versus membrane protein) and subcellular

214 location. We added transcript abundance as a covariate to the model, as the compensatory effect can

215 be expected to become larger at higher differential transcript levels. 


\section{Results}

\section{Aneuploidy globally impacts transcriptome and proteome of affected chromosomes}

We examined the impact of aneuploidy in L. donovani on transcriptome and proteome by integrated multi-omic analysis of 6 L. donovani strains from India and Nepal (Suppl. Table S1.A). These closely related strains are almost identical at the sequence level but differ greatly in their aneuploidy ${ }^{44}$. Specifically, the entire genome between any two strains of this set differs at most by 64 nonsynonymous SNPs and one indel in coding regions (Suppl. Table. S1.B). Thus, they are attractive model strains for studying the impact of aneuploidy on transcriptome and proteome with minimal interference of DNA sequence variation.

Genome, transcriptome, and proteome were obtained from the replicative promastigote stage (LOG) of the parasite. All genomes reached an average sequencing coverage of at least 26.5 fold, and $97.9 \%$ of the sequenced sites reached $10 \mathrm{X}$ coverage or more. Transcript samples had on average 4.71 million reads and obtained an average mapping efficiency of $97 \%$. Detailed mapping and coverage statistics for both genome and transcriptome data are available in Suppl. Table S1.C/S1.D. Identified indels and and estimations of chromosomal somy (i.e., chromosome copy number) and dosage of each gene in Suppl. Table S2. Identified transcripts and their counts are available in Suppl. Table S3. Proteome analysis identified in total 3145 proteins across all samples (Suppl. Table S4).

Our 6 L. donovani strains showed high but varying degrees of aneuploidy (Fig 1A). The most aneuploid strain was BPK288 with 12 aneuploid chromosomes, followed by BPK275 (10), BPK173 (10), BHU575 (10), BPK178 (9), and BPK282 (6). In L. donovani, chromosomes are numbered by increasing size. The baseline somy of chromosomes in L. donovani is $2 \mathrm{~N}$, except for chromosome 31 , which is always $4 \mathrm{~N}$ or more. Smaller chromosomes were more frequently aneuploid (Ld01, Ld05-Ld09, Ld11-Ld16), than larger chromosomes (Ld20, Ld23, Ld26, Ld31, Ld33, Ld35). Chromosome Ld31 was tetrasomic in all strains, and chromosome Ld23 always trisomic. Other aneuploid chromosomes had a variable somy. In bulk genomic analysis, somy values represent the average of the somy of individual cells present in a given population. Because of mosaicism, i.e. the presence of different kayotypes among individual cells of the parasite population in culture, many somy values were not discrete. The highest somy values (apart from Ld31) were observed for chromosomes Ld02 (somy = 3.9), Ld08 (3.9), and Ld33 (3.5) in BPK275, and for chromosome Ld05 (3.6) in BPK288. 
The transcript abundance of aneuploid chromosomes mirrored almost perfectly their underlying somy

247 (Fig 1B, Suppl. Fig S1A, S1B). Specifically, the average abundance of a transcript encoded by a

248 chromosome was proportional with a factor of 0.94 with the chromosomal somy (Fig 1C: $95 \% \mathrm{Cl}: 0.92$

$249-0.96$ and $\left.p<2 \cdot 10^{-16}, R^{2}: 0.98\right)$. The average abundance of the proteins encoded by a chromosome was also highly proportional to the aneuploidy of the chromosome $\left(p<2 \cdot 10^{-16}\right)$, albeit with a decreased slope of $0.74\left(95 \% \mathrm{Cl}: 0.69-0.76, \mathrm{R}^{2}: 0.90\right)$. Thus, aneuploidy results in a linear, but not equivalent, increase in protein abundance. Circos plots clearly showed that transcript- and protein abundance of aneuploid chromosomes was affected globally and not restricted to specific chromosomal regions (Fig 1B, Suppl. Fig S1A, S1B).

255 Interestingly, chromosome 35 exhibited only a partial amplification in strain BPK275, the first part 256 being disomic (somy $=2.2$ ) and the second part, starting from gene LdBPK_350040900, being trisomic (somy = 3.1) (Fig 1B). This phenomenon of partial chromosomal amplification has previously been observed for chromosome 23 as well ${ }^{45,46}$. The difference in both average transcript and protein abundance between the first and the second chromosome part was significant (t-test $p$ Trans $=4.0 \times 10^{-}$ more protein abundance. This demonstrates that also partial chromosomal amplifications affect transcript and protein abundance proportionally in the amplified region. 

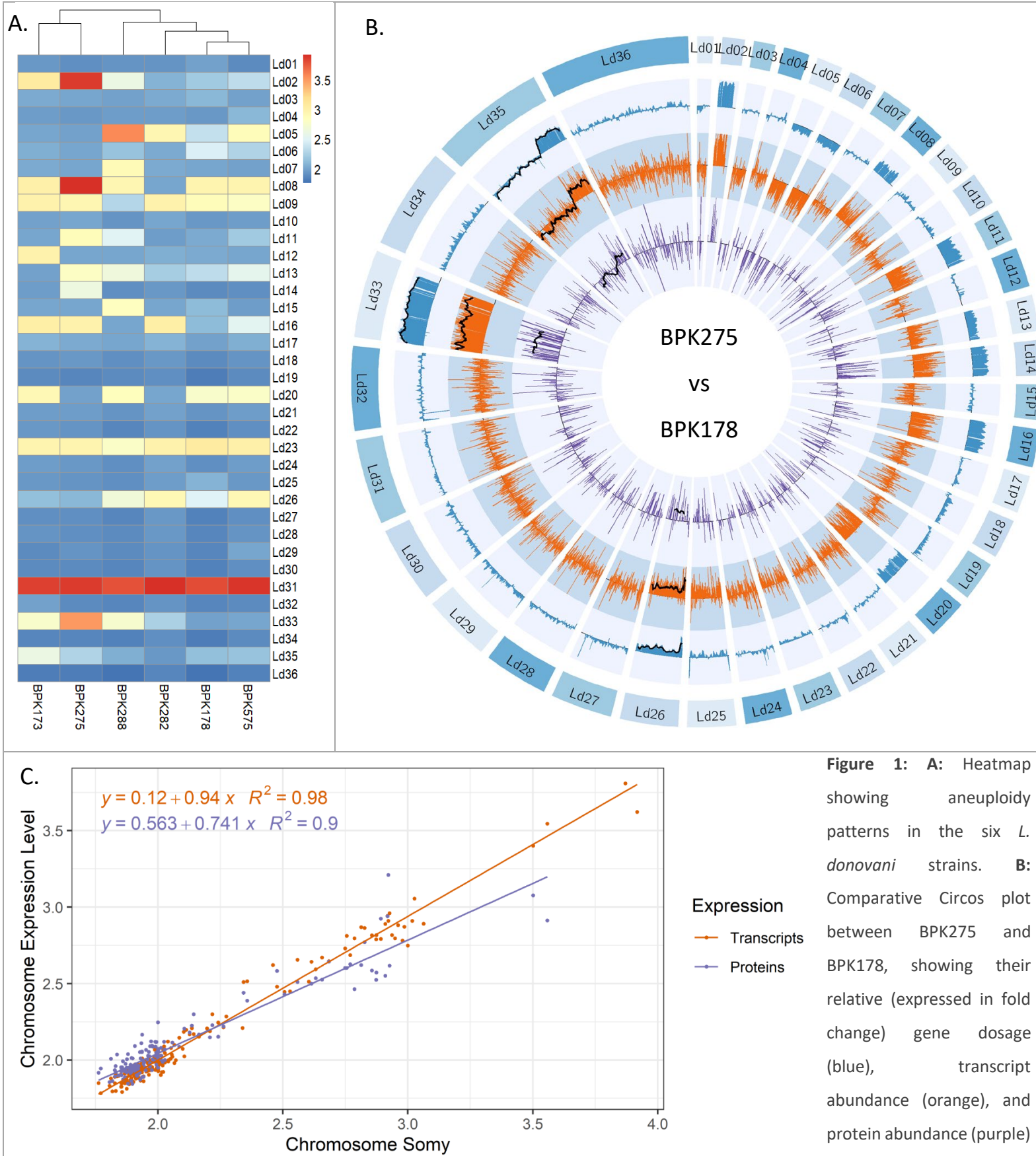

across the 36 L. donovani chromosomes. 20 point moving averages are indicated with a black line for the three largest aneuploid chromosomes. We show this strain pair because of the partial chromosomal amplification on chromosome Ld35. However, Circos plots between BPK173 and BPK282, and BHU575 and BPK288 show similar agreement between genome, transcriptome, and proteome and can be found in supplementary figures (Suppl. Fig S1A, S1B). C: Impact of chromosome aneuploidy on average chromosome transcript (red) and protein (blue) abundance levels. Each dot represents 1 chromosome in one of the six studied aneuploid L. donovani strains in the replicateive (LOG) phase. Abundance levels were normalized so that two transcript/protein abundance units match the abundance level of a disomic chromosome. Somy values are often not discrete due to mosaic aneuploidy (i.e. presence of different kayotypes among individual cells present in 1 strain) in Leishmania. 


\section{Aneuploidy does not impact all proteins equally: dosage compensation in Leishmania}

Our observation that a somy increase does not lead to an equivalent increase in protein abundance, but only $0.74 x$ the somy change, suggested either of two possible explanations. A first possibility is that all proteins encoded by an aneuploid chromosome are modulated equally and exhibit only a partial protein abundance increase. We further refer to this explanation as the 'dampening hypothesis'. Such a general 'dampening' effect would suggest an underlying process related to intrinsic mechanisms or limitations in the parasite's translation system. Alternatively, different proteins could respond differently to increased gene dosage and transcript abundance, pointing to compensatory effects linked to the identity, function, and physicochemical properties of those specific proteins or certain functional protein groups. We refer to this explanation as the 'compensation hypothesis'.

To evaluate both hypotheses, the differential abundance patterns of proteins were compared to their encoding chromosome differences from disomy to trisomy and tetrasomy. Suppose all proteins of a chromosome are affected equally by a shift in somy, following the 'dampening hypothesis'. In this case, the average of the protein abundance distribution should increase with increased somy, but the distribution shape should remain unaltered. In our study, three chromosomes (Ld05, Ld08, and Ld33) were at least once disomic, trisomic, and tetrasomic in one of our six study strains (Fig 1B), allowing for such comparative analysis. Ld02 also matched these criteria but was excluded from the analysis as only nine Ld02 proteins were detected. For reference, the same analysis was conducted for transcripts.

The average abundance of transcripts increased with somy, and the shape of the abundance distribution was found to be similar (Fig 2A). Specifically, the transcript abundance standard deviation did not significantly change with somy (Regression $p: 0.10$, Fig $2 \mathrm{~B}$ ), and the distributions were symmetric. This suggests that a somy change affects most transcripts equally, and dosage compensation at the transcript level is absent. Chromosome Ld08 had a higher variance in transcript abundance than the two other chromosomes. Our data is not conclusive as to whether this is due to the shift in somy or due to an intrinsically higher transcript abundance variance for this chromosome.

The average abundance of proteins also increased with somy, but the distribution shape was not consistent. This was particularly evident when comparing the disomic versus the tetrasomic chromosomes. We observed a clear left-tailing of the protein abundance distribution on chromosomes Ld08 and Ld33 chromosomes towards the lower, more conservative fold change values. Thus, while a subset of proteins follows the aneuploidy-induced gene dosage changes, another subset is maintained 
bioRxiv preprint doi: https://doi.org/10.1101/2021.09.14.460245; this version posted October 14, 2021. The copyright holder for this preprint (which was not certified by peer review) is the author/funder, who has granted bioRxiv a license to display the preprint in perpetuity. It is made available under aCC-BY-ND 4.0 International license.

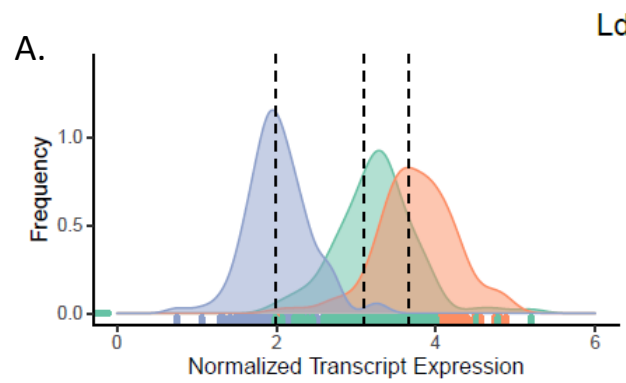

Ld05
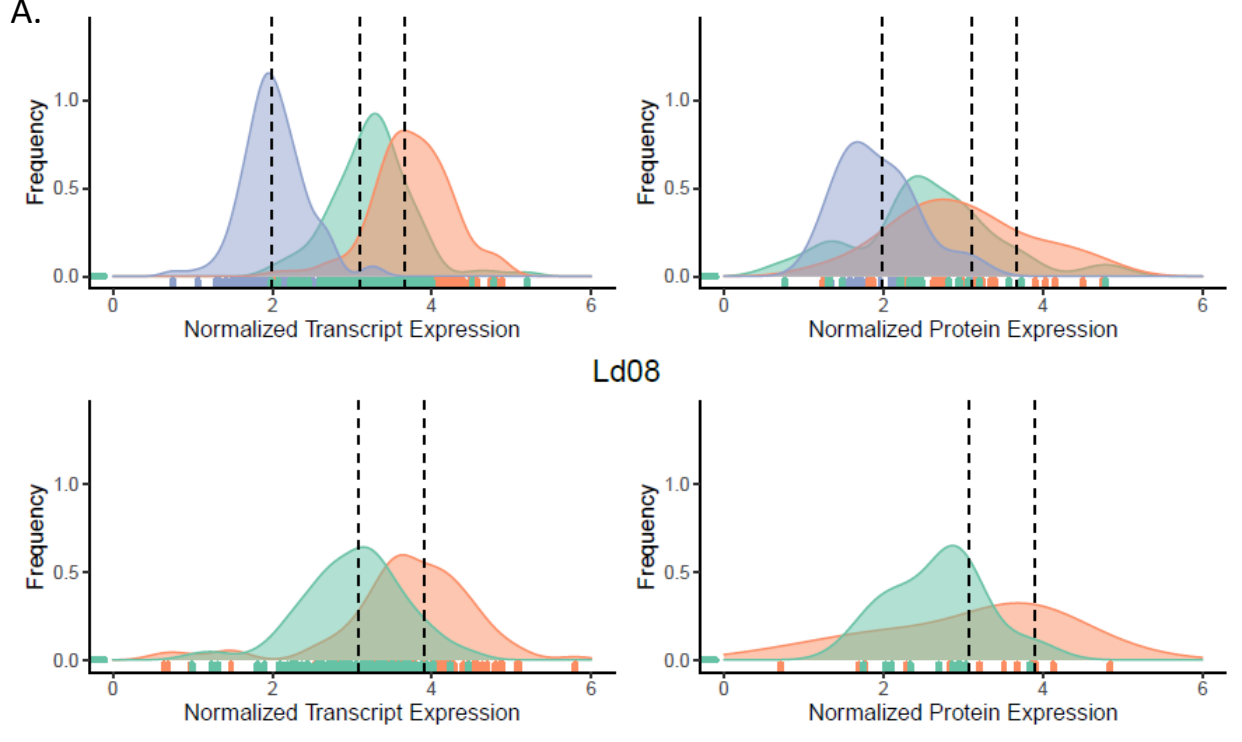

Ld08
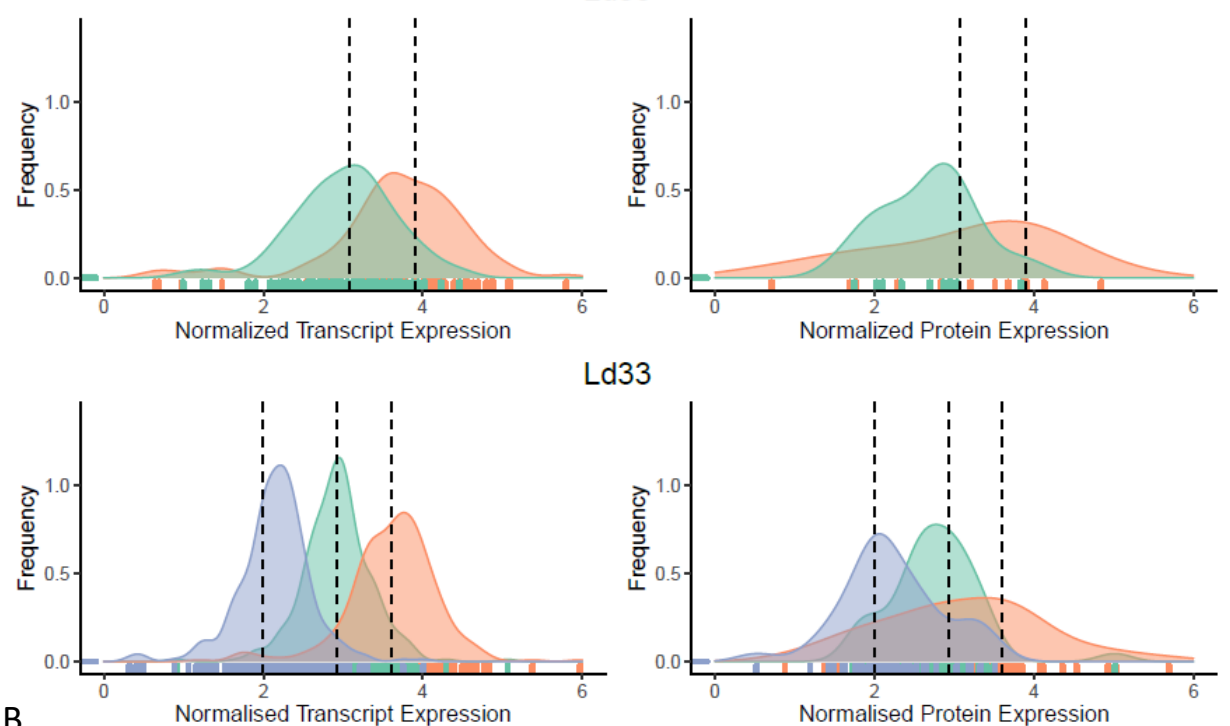

B.

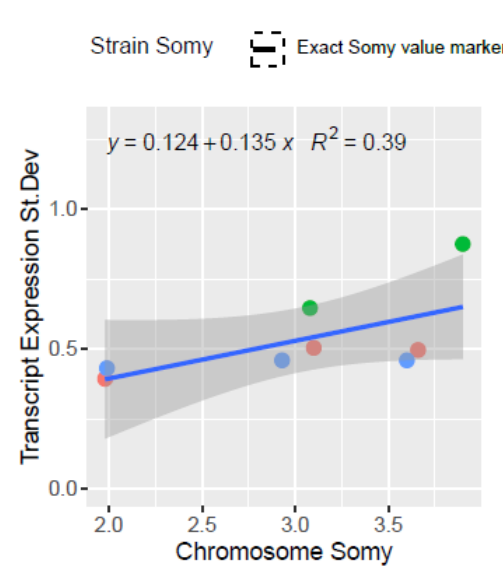

$\operatorname{Disomic}(\mathrm{N}=2) \quad \square$ Trisomic $(\mathrm{N}=3) \square$ Tetrasomic $(\mathrm{N}=3.6-4)$
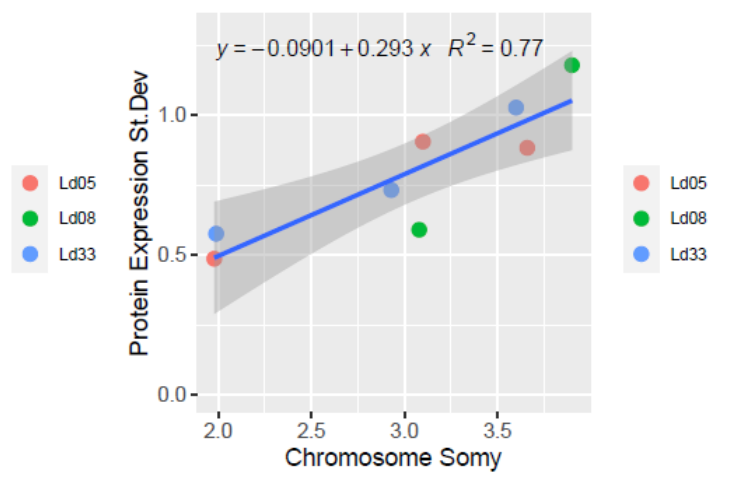

Figure 2: A: Normalised transcript (left) and protein (right) abundance patterns of disomic Ld05, Ld08, and Ld33 in their disomic (excl. chr 8), trisomic and tetrasomic state. Vertical dotted lines indicate the corresponding somy, derived from the genomic data. The small vertical lines at the base of the plot indicate individual datapoints for transcripts and proteins, from which the distributions were generated. The abundance of transcripts and proteins was normalized to their abundance in another strain that was disomic for their encoding chromosome (normalized abundance at disomy =2). This is the reason why we do not have disomic distributions for Ld08 since Ld08 was exclusively disomic in BPK282. B. Regression between the somy and transcript (left) or protein (right) standard deviation for chromosomes 
Compensated proteins are primarily protein complex subunits, secreted or non-

We investigated if specific protein properties were associated with the dosage compensation observed in our dataset. For this analysis, we defined a protein's compensation level as its $\log _{2}$ transcript fold change (i.e., its transcript level on the aneuploid chromosome divided by its transcript level on disomic chromosome), minus its $\log _{2}$ protein fold change. A particular challenge in this analysis was that protein fold changes, and hence, the observed compensation levels, were generally very small and close to measurement noise levels. For example, for a fully compensated protein from a trisomic chromosome, the compensation level would only be $0.5 \log _{2} \mathrm{FC}$ units ( $\log _{2} \mathrm{FC}$ transcript $\approx 0.5, \log _{2} \mathrm{FC}$ protein $=0$ ). Therefore, we focused on a set of 85 transcripts and proteins, which had very accurate protein measurements (SD between biological replicates $<0.25 \log _{2} \mathrm{FC}$ units). These covered a broad range of compensation levels ranging from $1.18 \log _{2} \mathrm{FC}$ units, to values close to zero, or even some proteins that had a slight overamplification compared to the transcript level (-0.40 $\log _{2} \mathrm{FC}$ units) (Suppl. Table S1.E).

We noted that within the top 5 most compensated proteins in this high-accuracy set, 3 coded for subunits of protein complexes: e.g., the cleavage and Polyadenylation Specificity Factor (CPSF) subunit A, the dynein light chain, and a Qc-SNARE protein had respectively 1.12, 0.96 , and 0.77 Log2FC units lower protein than transcript abundance. Accordingly, we hypothesized that protein complexes might be more likely to be dosage compensated. To test this systematically, all 85 proteins were screened for their involvement in heteromeric protein complexes. 47 proteins could be annotated (subunit/not a subunit) of which 16 were compensated ( $>=0.30$ Log2FC units), and 31 not ( $<0.30$ Log2FC units) (Suppl. Table S1.E). Strikingly, complex subunits accounted for $50.0 \%$ (8 out of 16) of the compensated proteins which was significantly more than the $6.5 \%$ ( 2 out of 31 ) we observed for the uncompensated proteins ( $\mathrm{Chi}^{2} p=0.00034$ ). For the majority of compensated subunits (6 out of 8 ), we could identify another subunit from the same complex, located on a different chromosome.

Next, we looked if there was a relation between dosage compensation and a set of biological and physicochemical protein properties to identify more subtle patterns. The following protein properties were checked for their relationship with protein dosage compensation using linear regression: the number of protein interactions, length (a proxy for transcript and protein size), hydrophobicity, membrane protein (yes/no), and subcellular location. Transcript abundance was added as a covariate to the model, as dosage compensation was larger at higher differential transcript levels $\left(p=5.5 \times 10^{-4}\right)$. 
The degree of dosage compensation of a protein was dependent on its cellular compartement (Suppl. Table S1.E. Cytoplasmatic proteins $(n=39)$ were significantly less compensated compared to all other proteins $(p=0.0054)$. Thus, cytoplasmatic proteins follow aneuploidy-induced dosage changes more closely than proteins from other cellular compartments. In contrast, proteins associated with the Golgi apparatus ( $n=2)$ had a significantly higher compensation than all other proteins ( $p=0.048)$. These two proteins were a vesicle-associated membrane protein and a small GTP-binding protein Rab18, both known to be secreted. Proteins from mitochondria $(n=18)$, nucleus $(n=13)$, peroxisome $(n=6)$ and other $(n=5)$ did not have significantly different compensation rates, and hence showed an intermediate level of dosage compensation. Other tested protein properties did not show a significant relation with dosage compensation.

\section{The impact of aneuploid chromosomes on the gene products of their euploid}

\section{counterparts}

In the previous sections, we reported how the aneuploidy of a chromosome affects the abundance of its encoded transcripts and proteins. However, through perturbation of regulatory networks, these changes might also affect transcripts and proteins encoded by genomic regions unaffected by aneuploidy. These were previously defined as 'trans-effects' and have been observed on the transcriptional level in diverse species, including humans, yeast, maize, and Arabidopsis ${ }^{47-50}$. Here we investigated the extend of trans-effects in Leishmania by comparing and characterizing the fractions of differential transcripts and proteins coming from aneuploid chromosomes, i.e. primary or cistranscripts and cis-proteins ${ }^{50}$, versus those coming from euploid chromosomes, i.e. trans-transcripts and trans-proteins.

We carried out differential transcript and protein abundance analyses (FDR adjusted $p<0.05$ ) between all possible pairs of the six L. donovani study strains (Table 1). On average, $71.8 \%$ of differentially expressed transcripts were cis-transcripts, while cis-proteins constituted only $40.7 \%$. The remaining $28.2 \%$ of differentially expressed transcripts and $59.3 \%$ of proteins were thus encoded by euploid chromosomes (trans-) and have therefore been regulated by another mechanism than gene dosage. Interestingly, only $20.0 \%$ of these trans-proteins had a corresponding transcript change ( $\log 2 \mathrm{FC} \mid>$ $0.5)$, indicating the vast majority is regulated at protein level directly and not through modulation of mRNA levels. Importantly, none of these trans-transcripts nor trans-proteins were associated with local copy number variants: these were rare and excluded from this analysis. Hence, the most probable explanation is that aneuploidy induces direct changes to cis-transcripts and cis-proteins, which in their 
turn directly (e.g., protein-protein interaction) or indirectly drive abundance changes in transtranscripts and mostly, trans-proteins.

To investigate this hypothesis further, we looked deeper into the functions of trans-transcripts and proteins. After pooling the trans-transcripts and trans-proteins from all comparisons, we identified a set of 307 unique trans-transcripts and 180 unique trans-proteins (duplicates and hypothetical proteins removed, Suppl. Tables S1.G \& S1.H). Overrepresentation analysis showed that no GO terms were significantly enriched in the pooled list of trans-transcripts or trans-proteins from all comparisons (FDR adjusted $p$ threshold 0.05), but it must be noted here that GO annotations in Leishmania are very poor due to its distinct evolutionary position compared to better studied model organisms. Only $48.7 \%$ of the L. donovani genes have at least one GO term, but they are often of the highest hierarchy (unspecific). Strikingly, $12.2 \%$ (22/180) of all trans-proteins concerned subunits of macromolecular complexes. A striking $82 \%(18 / 22)$ of these subunits had at least 1 other subunit located on an aneuploid chromosome. This suggests that subunit trans-proteins are a result of cis-protein subunits that alter the stability of all members of the macromolecular complex. Further, we found many transproteins to be involved in protein metabolism, transport and metabolic functions.

DIFFERENTIAL TRANSCRIPTS

\begin{tabular}{c|cccccc}
\hline $\begin{array}{c}\text { STRAIN } \\
\text { COMPARISON }\end{array}$ & Cis & Trans & N & Cis & Trans & N \\
275vs178 & 79.8 & 20.2 & 386 & 44.8 & 55.2 & 105 \\
\hline 275vs173 & 86.6 & 13.4 & 134 & 40.7 & 59.3 & 91 \\
275vs282 & 77.6 & 22.4 & 67 & 46.4 & 53.6 & 84 \\
288vs282 & 60.9 & 39.1 & 23 & 39.3 & 60.7 & 61 \\
275vs575 & 84.8 & 15.2 & 145 & 58.9 & 41.1 & 56 \\
173vs288 & 86.3 & 13.8 & 80 & 55.6 & 44.4 & 27 \\
173vs282 & 74.4 & 25.6 & 39 & 48.1 & 51.9 & 27 \\
173vs178 & 55.0 & 45.0 & 40 & 26.3 & 73.7 & 19 \\
575vs282 & 41.2 & 58.8 & 34 & 6.3 & 93.8 & 16 \\
\hline AVERAGE & 71.8 & 28.2 & & & & \\
\hline
\end{tabular}




\section{Aneuploidy globally affects transcriptome and proteome regardless of life stage}

So far, this study discussed results from the logarithmic growth stage (LOG), which essentially consists of Leishmania procyclic promastigotes (replicating, non-infectious). However, despite the absence of transcriptional regulation of individual protein-coding genes, transcripts and proteins can undergo post-transcriptional regulation during parasite differentiation. This raised the question whether the correlation between chromosome somy, average transcript abundance, and average protein abundance is also present in other life stages. Hence, for three strains, we also included the stationary growth phase (STAT). STAT predominantly consists of metacyclic promastigotes, which are nonreplicating, have a distinct morphology, are metabolically different, and infectious to the human host ${ }^{51}$. This was validated with metacyclogenesis markers META1 and HASPB, which were significantly upregulated in STAT as they are expected to be (Adj. $p<10^{-13}$, Suppl. Table 1.H). Transcriptome and proteome measurements and analysis were carried out exactly as for the LOG phase.

For both LOG and STAT, a chromosome's somy was proportional to its average transcript expression level, and protein expression level (Fig 3, $p<2 * 10^{-16}$ for transcript and protein in both LOG and STAT). The smaller number of data points led to slightly less stringent correlations than in our six strain analysis for LOG (Fig 1). Dosage compensation was apparent at the protein level, in both LOG and STAT growth phases. This suggests that the gene dosage changes associated with aneuploidy remain consistent throughout the parasite's life cycle.

A.

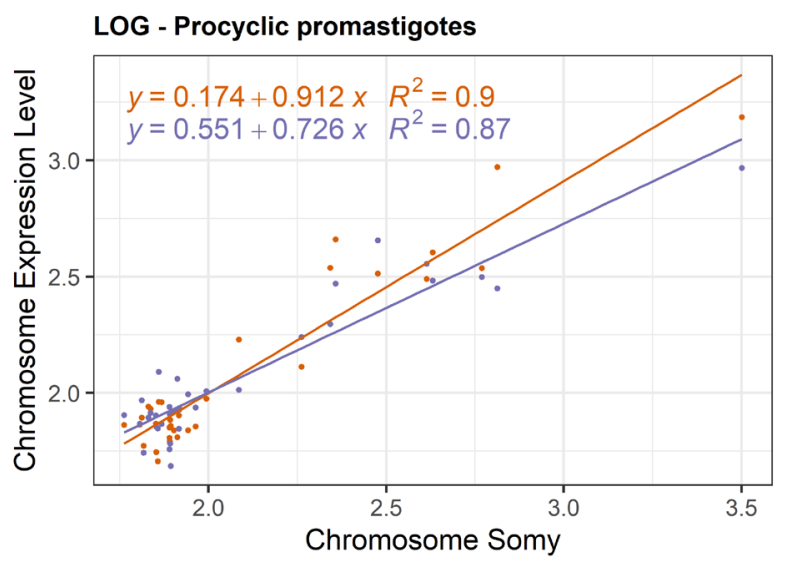

B.

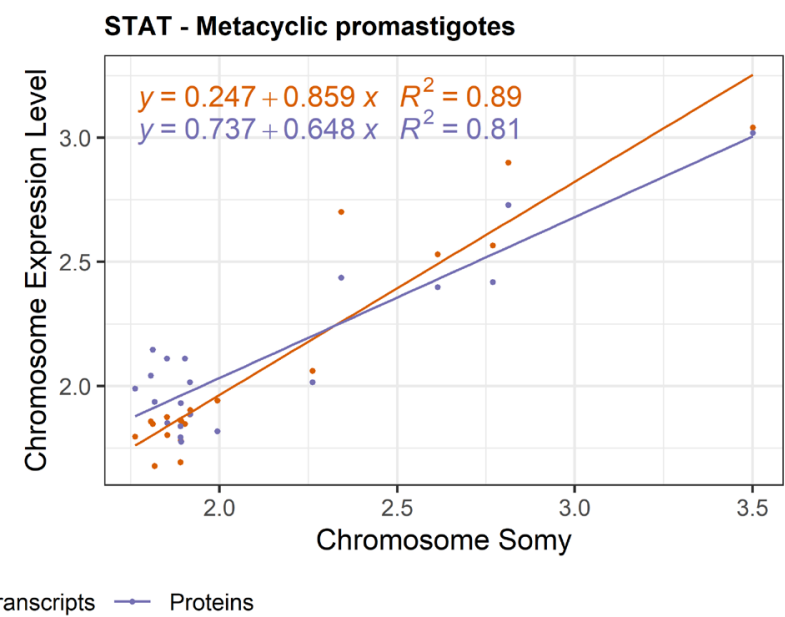




\section{Aneuploidy results in metabolic differences between $L$. donovani strains}

Among both cis- and trans-proteins we observed many responsible for metabolic functions. Thus, we ultimately aimed to understand if aneuploidy in L. donovani is reflected in the parasite's metabolome. Therefore, we carried out LC-MS metabolomics and confidently identified 78 metabolites across the six aneuploid study strains (Suppl. Table S5). In contrast to transcripts and proteins, there is no straightforward relation between a metabolite and a specific gene. Genes might affect metabolites indirectly, affect multiple metabolites, or affect no metabolite at all. Therefore, we turned to an indirect strategy to integrate the metabolomic data with our previous observations.

We determined if differences in aneuploidy between strains correlate with their metabolic differences. As such, we calculated the pairwise genomic (somy), transcriptomic, proteomic, and metabolic Euclidean distance between each possible strain pair, and correlated these distances (Fig 4). The larger the distance between these 2 strains, the more different their respective genomes, transcriptomes, proteomes, or metabolomes are from one another. Consistent with our other analyses, we found strong correlations between the genomic and transcriptomic distance, between the transcriptomic and proteomic distance, and between the genomic and proteomic distance ( $p \leq 0.001$ for all comparisons). However, the metabolome initially did not show any correlation with genome, transcriptome, and proteome. We suspected this might be caused by the high intrinsic levels of random variation of a subset of metabolites (by either technical or biological causes). Therefore, in the second phase of the analysis, we filtered on metabolites that had at least once a significant fold change (adjusted $p<0.05$ ) between two strains. This strategy selects only metabolites that are stable enough to reflect true differences in metabolite levels between strains. The resulting 'differentiating metabolome' consisting of 34 metabolites correlated strongly with all omic layers ( $p<0.05$, Fig 4 ). This demonstrates that the degree of karyotype difference between two strains correlates with their degree of metabolic differences. Specifically, the differentiating metabolome included 9 amino acids (alanine, aspartate, cysteine, leucine/isoleucine (not distinguishable), methionine, phenylalanine, tryptophan, tyrosine, and valine), suggesting the amino acid metabolism is an important molecular difference between these aneuploid parasites. 
bioRxiv preprint doi: https://doi.org/10.1101/2021.09.14.460245; this version posted October 14 2021. The copyright holder for this preprint (which was not certified by peer review) is the author/funder, who has granted bioRxiv a license to display the preprint in perpetuity. It is made available under aCC-BY-ND 4.0 International license.

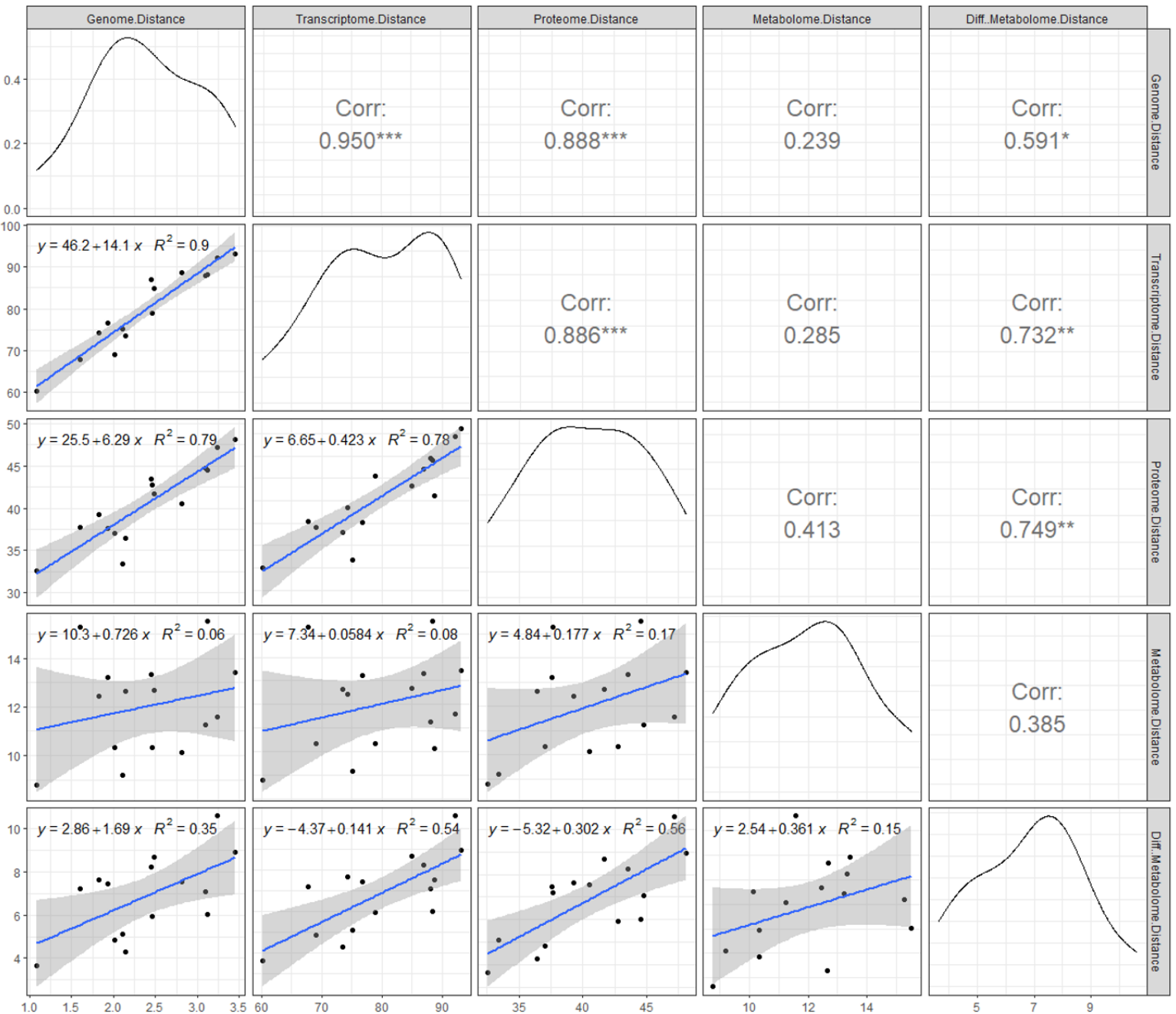

Figure 4: How differences between two L. donovani strains on 1 'omic layer correlate with their differences on another omic layer. The plots show the pairwise correlations between the genome distance (aneuploidy), transcriptome distance, proteome distance, metabolome distance (all 78 metabolites), and differentiating metabolome distance (34 metabolites), each time calculated between 2 L. donovani strains. Each dot represents 1 comparison between two strains. With 6 strains in our experiment, there were 15 possible pairwise comparisons. The distance metric that was used is the Euclidean distance. Somy values were used directly, but transcript abundance, protein abundance or metabolite abundance were first Z-scored before calculating the distance. The top-right panels show the Pearson correlation coefficients with $*=$ Pval $<0.05, * *$ Pval $<0.01, * * *=$ Pval $<0.001$, while the bottom-left panels show the linear regressions. 


\section{Discussion}

447

448

449

450

451

452

453

454

455

456

457

458

459

460

461

462

463

464

465

466

467

468

469

470

471

472

473

474

475

476

Leishmania displays a remarkably high tolerance for aneuploidy, with any chromosome having the capacity to become polysomic and produce a viable parasite. The high incidence of aneuploidy in experimental conditions stresses its central importance for the parasite to adapt to novel environments ${ }^{10}$. However, it remained unclear how this large genomic disruption affects the parasite as a system. Moreover, Leishmania's distinct phylogenetic status and unique gene expression system disallow extrapolation from model systems. Therefore, we investigated the systemic impact of aneuploidy in L. donovani by integrating, for the first time, genome, transcriptome, proteome, and metabolome profiling data of highly aneuploid strains. Our study offers a comprehensive picture of the molecular changes associated with aneuploidy in this unique Eukaryotic system that lacks transcriptional regulation of individual protein-coding genes.

We confirmed that the transcript abundance of aneuploid chromosomes is globally affected. This observation is in line with previous work that has also found a strong link between aneuploidy-induced gene dosage changes and transcript abundance in Leishmania ${ }^{13,16,52}$. We further showed that this relation is almost equivalent, with transcription levels closely mirroring chromosome copy number changes (transcript abundance $=0.94 \mathrm{x}$ the somy). The impact of aneuploidy on the $L$. donovani proteome was also linear and chromosome-wide, but not equivalent. For every chromosome copy, the protein abundance levels of that chromosome increased (on average) by a factor $0.74 x$ the somy.

This reduced impact of gene dosage on the protein level was due to the compensation of a subset of proteins. While some proteins did follow the aneuploidy-induced gene dosage changes, the dosage of a subset was compensated. This compensation ranged from partial to complete compensation to disomic levels. Our data thus suggest that these proteins are somehow post-transcriptionally attenuated. We made two observations concerning the identity of compensated proteins that might explain this attenuation. Firstly, compensated proteins were enriched $(50.0 \%)$ for subunits of macromolecular protein complexes. This striking observation confirms several observations in aneuploid human- and yeast cell lines $47,48,53,54$ that also detected protein complex subunits to be highly prevalent amongst compensated proteins. It can be explained by the fact that individual subunits of macromolecular complexes are typically unstable unless assembled into a stable complex ${ }^{47}$. As subunits of a single complex are often encoded on different chromosomes and produced in balanced, stoichiometric amounts, an increase in an individual subunit (here: by aneuploidy) will often not lead to more complex formation ${ }^{47,48,55}$. Instead, the protein finds itself without binding partner(s), remains 
unstable, and rapidly degrades. Secondly, we observed a correlation between the degree of compensation and the cellular destination of the compensated protein. Two secreted proteins associated with the Golgi apparatus were the most compensated (i.e. their expression was close to disomic levels), which could be explained by the fact that secreted proteins do not accumulate in the cell. Correspondingly, dosage compensation of secreted proteins was also observed in a recent study of human fibroblasts with trisomy $21^{47}$. Proteins from mitochondria, the nucleus, and the peroxisome were also significantly more compensated than cytoplasmatic proteins, which had the lowest level of compensation (i.e., cytoplasmic proteins followed gene dosage the best). A potential explanation might be that mitochondrial, nucleic, and peroxisome proteins require active transport into their destined organelle. This transport requires extensive interaction with other proteins, potentially explaining why their abundance scales less with increased protein production. In aneuploid (disomic II) strains of S. cerevisiae, increased translation was found of genes related to protein trafficking pathways of the endomembrane system and nucleus, also suggesting dependency on this system ${ }^{56}$.

Partial chromosomal amplification can occur in Leishmania. We have previously observed this phenomenon for chromosome Ld23 ${ }^{45,46}$ and in the present study for Ld35. Interestingly, gene dosage proportionally affected transcript and protein abundance for these subchromosomal amplifications as well. This suggests that our findings can be extrapolated from aneuploidy to the concept of gene dosage in general. Further, we demonstrated that these gene dosage changes remain important throughout the life cycle of the parasite. Indeed, we observed an identical impact of gene dosage on transcriptome and proteome in either LOG (enriched for procyclic promastigotes, proliferative stage) or STAT (enriched for metacyclic promastigotes, infectious stage) growth phases. This is striking, as the parasite featuring extensive transcriptome and proteome changes during its life cycle. For example, transcriptomics studies have shown that up to $30-50 \%$ of transcripts are differentially expressed between procyclic and metacyclic promastigotes ${ }^{15,57}$. Similarly, $10-40 \%$ of proteins are differentially expressed between the parasite's promastigote and amastigote life stages ${ }^{13,58}$.

A limited proportion of differential transcripts between aneuploid strains $(23.6 \%)$ and the majority of differential proteins (56.9\%) originated from chromosomes without a change in somy, so-called transtranscripts, and trans-proteins. As we excluded the few genes with SNPs and INDELs from this analysis, this suggests that the molecular impact of aneuploidy in Leishmania extends beyond the genes encoded on the aneuploid chromosomes. It further appears that trans-protein abundances are modulated at the translation or protein-stability level, as the majority did not have underlying changes in transcript level. Interestingly, these results contrast with published findings for most other 
Eukaryotes. High abundances of trans-transcripts have been reported in several other aneuploidy studies on cells from humans, yeast, maize, and Arabidopsis ${ }^{47-50}$. These trans-transcriptomes were

511 typically enriched for transcription factors, genes related to metabolic processes, protein metabolism, or cellular stress responses. It supports the idea that the cellular system mediates the primary impact of aneuploidy by inducing transcriptional changes to trans-genes. We did not observe this coordinated transcriptomic response in Leishmania. This matches the constitutive gene abundance model of the parasite, where the transcription of individual protein-coding genes can not be controlled. Instead, we found a large proportion of trans-proteins, which might be modulated directly at the protein level. This modulation may also be present in other Eukaryotes, but could be overshadowed by the transcription factor-induced changes. For this reason, we propose Leishmania as an attractive model to study processes of post-transcriptional modulation of protein levels in the context of aneuploidy.

Finally, we showed that the degree of aneuploidy differences between strains matched the degree of transcriptome, proteome, and differential metabolome changes. This is an important observation as it univocally demonstrates that aneuploidy in Leishmania has the capacity to drive metabolic variation, which is closely linked to the phenotype. It also matches our previous observations where we linked $L$. donovani gene copy number variants directly to changes in metabolic pathways ${ }^{32}$. Ultimately, it supports the view that aneuploidy can be adaptive and drive the metabolic changes that the parasite needs to survive in a novel environment.

\section{7}

528

\section{Conclusion}

In summary, our study shows that aneuploidy in Leishmania globally and drastically impacts the transcriptome and proteome of affected chromosomes throughout the parasite's life cycle. Ultimately, these aneuploidy-induced changes can drive metabolomic differences between Leishmania strains. Similarly as in other Eukaryotes, transcripts and proteins follow gene dosage, with the notable exception of macromolecular subunits and secreted proteins. While most aneuploid Eukaryotes display extensive transcriptional regulation of trans-transcripts to mediate the effects of aneuploidy, we did not observe this in Leishmania. Instead, Leishmania features a surprisingly high number of trans-proteins, which are likely post-transcriptionally modulated. This modulation may also be present in other Eukaryotes, but could be overshadowed by the transcription factor-induced gene regulation that is absent in Leishmania. For this reason, we believe that Leishmania is an attractive model to study processes of post-transcriptional modulation of protein levels in the context of aneuploidy and beyond. 


\section{0}

\section{References}

5411 Siegel, J. J. \& Amon, A. New Insights into the Troubles of Aneuploidy. Annual review of cell and

542 developmental biology 28, 189-214, doi:10.1146/annurev-cellbio-101011-155807 (2012).

5432 Zhu, J., Tsai, H.-J., Gordon, M. R. \& Li, R. Cellular Stress Associated with Aneuploidy. Dev Cell

544

545 44, 420-431, doi:10.1016/j.devcel.2018.02.002 (2018). adaptation through induction of aneuploidy. Nature 482, 246, doi:10.1038/nature10795 (2012).

4 Selmecki, A., Forche, A. \& Berman, J. Genomic plasticity of the human fungal pathogen Candida albicans. Eukaryotic cell 9, 991-1008, doi:10.1128/ec.00060-10 (2010).

5 Gresham, D. et al. The repertoire and dynamics of evolutionary adaptations to controlled nutrient-limited environments in yeast. PLoS genetics 4, e1000303, doi:10.1371/journal.pgen.1000303 (2008).

6 Gerstein, A. C. et al. Polyploid titan cells produce haploid and aneuploid progeny to promote stress adaptation. mBio 6, e01340-01315, doi:10.1128/mBio.01340-15 (2015).

7 Seton-Rogers, S. Fitness penalties of aneuploidy. Nature Reviews Cancer 17, 142-143, doi:10.1038/nrc.2017.9 (2017).

8 Rancati, G. et al. Aneuploidy underlies rapid adaptive evolution of yeast cells deprived of a conserved cytokinesis motor. Cell 135, 879-893, doi:10.1016/j.cell.2008.09.039 (2008).

560

561

562

563

564

565

566

567

568

569

570

571

572

573

Sunshine, A. B. et al. The Fitness Consequences of Aneuploidy Are Driven by ConditionDependent Gene Effects. PLOS Biology 13, e1002155, doi:10.1371/journal.pbio.1002155 (2015).

10 Imamura, H. et al. Evolutionary genomics of epidemic visceral leishmaniasis in the Indian subcontinent. eLife 5, e12613 (2016).

11 Franssen, S. U. et al. Global genome diversity of the Leishmania donovani complex. eLife 9, e51243, doi:10.7554/eLife.51243 (2020).

12 Negreira, G. H. et al. Exploring the evolution and adaptive role of mosaic aneuploidy in a clonal Leishmania donovani population using high throughput single cell genome sequencing. bioRxiv, 2020.2003.2005.976233, doi:10.1101/2020.03.05.976233 (2020).

13 Dumetz, F. et al. Modulation of Aneuploidy in Leishmania donovani during Adaptation to Different In Vitro and In Vivo Environments and Its Impact on Gene Expression. mBio 8, e00599-00517 (2017).

14 Ivens, A. C. et al. The genome of the kinetoplastid parasite, Leishmania major. Science (New York, N.Y.) 309, 436-442, doi:10.1126/science.1112680 (2005).

15 Cuypers, B. et al. Multiplexed Spliced-Leader Sequencing: A high-throughput, selective method for RNA-seq in Trypanosomatids. Scientific Reports 7, 3725, doi:10.1038/s41598-017-03987-0 (2017).

16 Prieto Barja, P. et al. Haplotype selection as an adaptive mechanism in the protozoan pathogen Leishmania donovani. Nature ecology \& evolution, doi:10.1038/s41559-017-0361-x (2017).

17 WHO. Proposals for the nomenclature of salivarian trypanosomes and for the maintenance of reference collections*. Bulletin of the World Health Organization 56, 467-480 (1978).

18 Andrews, S. FastQC: a quality control tool for high throughput sequence data. (2010).

19 Bolger, A. M., Lohse, M. \& Usadel, B. Trimmomatic: a flexible trimmer for Illumina sequence data. Bioinformatics (Oxford, England) 30, 2114-2120, doi:10.1093/bioinformatics/btu170 (2014). 
58520 Dumetz, F. et al. Modulation of Aneuploidy in Leishmania donovani during Adaptation to Different In Vitro and In Vivo Environments and Its Impact on Gene Expression. mBio 8, doi:10.1128/mBio.00599-17 (2017).

$21 \mathrm{Li}, \mathrm{H}$. Aligning sequence reads, clone sequences and assembly contigs with BWA-MEM. arXiv preprint arXiv:1303.3997 (2013).

22 Li, H. A statistical framework for SNP calling, mutation discovery, association mapping and population genetical parameter estimation from sequencing data. Bioinformatics (Oxford, England) 27, 2987-2993, doi:10.1093/bioinformatics/btr509 (2011).

23 Downing, T. et al. Whole genome sequencing of multiple Leishmania donovani clinical isolates provides insights into population structure and mechanisms of drug resistance. Genome research 21, 2143-2156, doi:10.1101/gr.123430.111 (2011).

24 Anders, S., Pyl, P. T. \& Huber, W. HTSeq--a Python framework to work with high-throughput sequencing data. Bioinformatics (Oxford, England) 31, 166-169, doi:10.1093/bioinformatics/btu638 (2015).

25 Quinn, T. P., Erb, I., Richardson, M. F. \& Crowley, T. M. Understanding sequencing data as compositions: an outlook and review. Bioinformatics (Oxford, England) 34, 2870-2878, doi:10.1093/bioinformatics/bty175 (2018).

601

Love, M. I., Huber, W. \& Anders, S. Moderated estimation of fold change and dispersion for RNA-seq data with DESeq2. Genome biology 15, 550, doi:10.1186/s13059-014-0550-8 (2014). Tyanova, S., Temu, T. \& Cox, J. The MaxQuant computational platform for mass spectrometrybased shotgun proteomics. Nature Protocols 11, 2301-2319, doi:10.1038/nprot.2016.136 (2016).

Berg, M. et al. Experimental resistance to drug combinations in Leishmania donovani: metabolic and phenotypic adaptations. Antimicrobial agents and chemotherapy 59, 2242-2255 (2015).

Berg, M. et al. Metabolic adaptations of Leishmania donovani in relation to differentiation, drug resistance, and drug pressure. Mol Microbiol 90, 428-442, doi:10.1111/mmi.12374 (2013).

31 Scheltema, R. A., Jankevics, A., Jansen, R. C., Swertz, M. A. \& Breitling, R. PeakML/mzMatch: a file format, Java library, $\mathrm{R}$ library, and tool-chain for mass spectrometry data analysis. Analytical chemistry 83, 2786-2793, doi:10.1021/ac2000994 (2011).

32 Cuypers, B. et al. Integrated genomic and metabolomic profiling of ISC1, an emerging Leishmania donovani population in the Indian subcontinent. Infection, Genetics and Evolution 62, 170-178, doi:10.1016/j.meegid.2018.04.021 (2018).

33 Prince, J. T. \& Marcotte, E. M. Chromatographic alignment of ESI-LC-MS proteomics data sets by ordered bijective interpolated warping. Anal Chem 78, 6140-6152, doi:10.1021/ac0605344 (2006).

Tautenhahn, R., Böttcher, C. \& Neumann, S. Highly sensitive feature detection for high resolution LC/MS. BMC Bioinformatics 9, 504, doi:10.1186/1471-2105-9-504 (2008).

5 Doyle, M. A. et al. LeishCyc: a biochemical pathways database for Leishmania major. BMC Syst Biol 3, 57-57, doi:10.1186/1752-0509-3-57 (2009).

Fahy, E. et al. Update of the LIPID MAPS comprehensive classification system for lipids. J Lipid Res 50 Suppl, S9-S14, doi:10.1194/jIr.R800095-JLR200 (2009). genomes, pathways, diseases and drugs. Nucleic acids research 45, D353-d361, doi:10.1093/nar/gkw1092 (2017). 
63438 Wishart, D. S. et al. HMDB 4.0: the human metabolome database for 2018. Nucleic acids research 46, D608-d617, doi:10.1093/nar/gkx1089 (2018).

39 Tihon, E. et al. Genomic analysis of Isometamidium Chloride resistance in Trypanosoma congolense. International Journal for Parasitology: Drugs and Drug Resistance 7, 350-361, doi:10.1016/j.ijpddr.2017.10.002 (2017).

40 Wickham, H. ggplot2: Elegant Graphics for Data Analysis. (Springer-Verlag New York, 2016).

64041 Krzywinski, M. I. et al. Circos: An information aesthetic for comparative genomics. Genome research, doi:10.1101/gr.092759.109 (2009).

643

42 Hunter, J. D. Matplotlib: A 2D Graphics Environment. Computing in Science \& Engineering 9, 90-95, doi:10.1109/MCSE.2007.55 (2007).

43 Virtanen, P. et al. SciPy 1.0: fundamental algorithms for scientific computing in Python. Nature Methods 17, 261-272, doi:10.1038/s41592-019-0686-2 (2020).

647 Imamura, H. et al. Evolutionary genomics of epidemic visceral leishmaniasis in the Indian subcontinent. elife 5, doi:10.7554/eLife.12613 (2016).

649

Dumetz, F. et al. Molecular Preadaptation to Antimony Resistance in Leishmania donovani on the Indian Subcontinent. mSphere 3, e00548-00517, doi:10.1128/mSphere.00548-17 (2018).

46 Laffitte, M.-C. N. et al. Formation of Linear Amplicons with Inverted Duplications in Leishmania Requires the MRE11 Nuclease. PLoS genetics 10, e1004805, doi:10.1371/journal.pgen.1004805 (2014).

652

Hwang, S. et al. Consequences of aneuploidy in human fibroblasts with trisomy 21. Proceedings of the National Academy of Sciences 118, e2014723118, doi:10.1073/pnas.2014723118 (2021). Torres, E. M. et al. Effects of Aneuploidy on Cellular Physiology and
Yeast. Science 317, 916-924, doi:10.1126/science.1142210 (2007). Makarevitch, I., Phillips, R. L. \& Springer, N. M. Profiling expression changes caused by a segmental aneuploid in maize. BMC Genomics 9, 7, doi:10.1186/1471-2164-9-7 (2008). e1000226, doi:10.1371/journal.pgen.1000226 (2008). (1) B. Kreil, D. P., Matzke, M. \& Matzke, A. J. M. Effects of Aneuploidy on Genome Structure, Expression, and Interphase Organization in Arabidopsis thaliana. PLoS genetics 4,

51 Berg, M. et al. Metabolic adaptations of Leishmania donovani in relation to differentiation, drug resistance, and drug pressure. Molecular microbiology 90, 428-442, doi:10.1111/mmi.12374 (2013).

52 lantorno, S. A. et al. Gene Expression in Leishmania Is Regulated Predominantly by Gene Dosage. mBio 8, doi:10.1128/mBio.01393-17 (2017).

53 Dephoure, N. et al. Quantitative proteomic analysis reveals posttranslational responses to aneuploidy in yeast. elife 3, e03023, doi:10.7554/eLife.03023 (2014).

54 Stingele, S. et al. Global analysis of genome, transcriptome and proteome reveals the response to aneuploidy in human cells. Mol Syst Biol 8, 608, doi:10.1038/msb.2012.40 (2012).

55 Veitia, R. A., Bottani, S. \& Birchler, J. A. Cellular reactions to gene dosage imbalance: genomic, transcriptomic and proteomic effects. Trends in Genetics 24, 390-397, doi:10.1016/j.tig.2008.05.005 (2008).

Larrimore, K. E., Barattin-Voynova, N. S., Reid, D. W. \& Ng, D. T. W. Aneuploidy-induced proteotoxic stress can be effectively tolerated without dosage compensation, genetic mutations, or stress responses. BMC Biology 18, 117, doi:10.1186/s12915-020-00852-x (2020).

57 Dillon, L. A. L. et al. Transcriptomic profiling of gene expression and RNA processing during Leishmania major differentiation. Nucleic acids research 43, 6799-6813, doi:10.1093/nar/gkv656 (2015). 
58 Lynn, M. A., Marr, A. K. \& McMaster, W. R. Differential quantitative proteomic profiling of Leishmania infantum and Leishmania mexicana density gradient separated membranous fractions. J Proteomics 82, 179-192, doi:10.1016/j.jprot.2013.02.010 (2013).

\section{Funding}

This work was supported by the Research Foundation Flanders [1101614N to B.C.]; the Interuniversity Attraction Poles Program of Belgian Science Policy [P7/41 to JC.D.], the InBev Baillet-Latour foundation and the Department of Economy, Science and Innovation in Flanders [ITM-SOFIB, SINGLE to JC.D.]

\section{Acknowledgements}

We thank the Center of Medical Genetics at the University of Antwerp for hosting the NGS facility on which our RNA-Seq experiments were performed. The computational resources used for this work were provided by the VSC (Flemish Supercomputer Center) at the University of Antwerp.

\section{Data Availability Statement}

The raw genomic and transcriptomic data in this study is available at the NCBI Sequence Read Archive (SRA) under the accession code: PRJNA762444. Raw proteomic data has been submitted to the PRIDE database under reference 1-20210911-141001. Raw metabolomic data was submitted to the MetaboLights database under accession code MTBLS1612.

\section{Conflict of Interest}

The authors declare that they have no conflict of interest. 


\section{Supplementary Tables}

701

702

703

704

705

706

707

708

709

710

711

712

713

714

715

716

717

\section{Supplementary Table S1.A}

Detailed description of the L. donovani isolates used in this study.

\section{Supplementary Table S1.B}

Number of total SNPs, non-synonmous SNPs and homozygous non-synonymous SNPs between any pair of $L$. donovani strains in this study.

\section{Supplementary Table S1.C}

Sequencing quality and mapping statistics of all 6 sequenced L. donovani genomes in this study.

\section{Supplementary Table S1.D}

Sequencing quality and mapping statistics of all sequenced $L$. donovani transcriptomes in this study.

\section{Supplementary Table S1.E}

Dosage compensation model data, coefficients and $p$-values.

\section{Supplementary Table S1.F}

Summary of all cis- and trans- transcripts and proteins of all possible pairwise comparisons between the 6 L. donovani strains in this study.

\section{Supplementary Table S1.G}

Pooled trans-transcripts from all possible pairwise comparisons between the 6 L. donovani strains in this study.

\section{Supplementary Table S1.H}

Pooled trans-proteins from all possible pairwise comparisons between the 6 L. donovani strains in this study.

\section{Supplementary Table S1. I}

Upregulated metacyclogenis markers in STAT growth phase. Output generated by DESeq2.

\section{Supplementary Table S2}

Structure (Somy and local CNVs) of all sequenced $L$. donovani genomes in this study.

\section{Supplementary Table S3}

Differential RNA abundance analyses of the different biological comparisons made in this study. 


\section{Supplementary Table S4}

729 Detailed statistics about all proteins detected in this study. Output generated by MaxQuant.

\section{Supplementary Table S5}

731 Detailed statistics about all metabolites detected in this study.

\section{Supplementary Figures}

\section{Supplementary Figure S1.A \& S1.B}

734 Comparative Circos plot between BPK173 and BPK288 (S1.A) and between BHU575 and BPK282 (S1.B), showing their relative (expressed in fold change) gene dosage (blue), transcript abundance (orange), and protein abundance (purple) across the 36 L. donovani chromosomes. 